\title{
A Covid-19 Teaching Example: Pooled Testing with Microsoft Excel ${ }^{\circledR}$
}

\author{
Humberto Barreto \\ Professor of Economics and Management \\ DePauw University
}

August 25, 2020

\section{DePauw University Working Papers 2020-1}

\begin{abstract}
:
This paper uses pooled testing as a teaching example for undergraduate statistics, econometrics, or quantitative methods courses. It offers step-bystep instructions to create an Excel spreadsheet that uses Monte Carlo simulation to find the optimal group size for a given infection rate. A completed version of the spreadsheet, along with readings, questions, and analytical solution is available at tiny.cc/pooledtesting.
\end{abstract}

JEL Codes: A2, C1, I10

Keywords: coronavirus, Monte Carlo, simulation, optimization, spreadsheet, pedagogy 


\section{Note to Professor}

This paper uses pooled testing as a teaching example for undergraduate statistics, econometrics, or quantitative methods courses. Pooled testing is simple to explain, yet it has engaging aspects and questions. The covid-19 pandemic has made the randomness of getting infected tangible. Likewise, testing for disease is now commonly understood. In this case, the data generation process is easy to see and we can model it. Furthermore, we have a novel optimization problem on our hands - we want to find the group size that minimizes the total number of tests.

Starting from a blank Excel spreadsheet, the material is delivered via stepby-step instructions. It utilizes functions and tools that students are unlikely to have seen before, but are accessible to the typical undergraduate.

This paper offers instructions for creating the spreadsheet and explains basic concepts. There are several possible delivery modes for course use. A professor can display or share the screen while constructing the sheet, adding comments and answering questions. Or, the instructions in the pages that follow can be provided to students as a handout for use in a computer lab, online course, or assignment, with the professor consulting with students who need help. These can be combined in a hybrid version, where the professor does some of the work in class and leaves the rest for students to complete on their own.

The pages that follow can be extracted from this pdf and you may modify, delete or add text with a pdf editor such as Adobe Acrobat Pro. For more extensive edits or substantial modifications, the LaTex document is available at academic.depauw.edu/ hbarreto/working.

A completed version of the Excel file is available at the website above and via this direct link: tiny.cc/pooledtesting. It also includes readings, questions for discussion and further work, along with advanced material such as analytical solutions. 


\section{Introducing Pooled Testing}

Pooled testing is in the news because it can be used with covid-19 samples (Mandavilli, 2020). The basic idea, however, is quite general and pooled testing was, in fact, used by the United States military during WWII to test men for syphilis (Dorfman, 1943).

The logic of pooled testing is straightforward. A university, for example, could take saliva or nasal swab samples from each student and test them individually or it could combine parts of each sample into groups (say, five or ten) and test the pooled sample. If it is negative, then all of the individuals in that group are negative and we have saved on testing every person in that group. If the pooled sample is positive, then individual tests would be performed on the reserved parts of each individual's sample to determine exactly who is infected.

This leads to a crucial question: What is the optimal group size? The bigger the group, the lower the number of groups tested, but the higher the chances a group is positive and then everyone in the group has to be tested (we ignore the possibility of sub-group testing, false positives or negatives, and other complications).

We answer this question by constructing an Excel spreadsheet. We proceed step-by-step and reveal Excel functions and tools as we create our model of pooled testing.

\section{The Data Generation Process}

The first thing we need to do is implement the random process by which some people get infected and others do not. We do this by drawing a random number and comparing it to a threshold value so we get either a zero (not infected) or a one (infected).

We assume that everyone has the same likelihood of catching the virus, say $5 \%$. This is a parameter in our model and it will serve as our threshold value for determining if someone is infected.

STEP Enter 5\% in cell A1 of a blank spreadsheet and label it as "infection rate" in cell B1. Save the Excel file (PooledTesting is a good name). 
Cell A1 displays 5\%, which is the same as 0.05 in decimal notation. The number 0.05 is what the spreadsheet stores in its internal memory. It is worth remembering that what is displayed may be different from what is stored.

We can, of course, refer to cell A1, but cell addresses can be difficult to read. It is good practice to name a cell or a cell range so that formulas can use natural language to reference cells.

$S T E P$ Name cell A1 InfectionRate because this will make our future formulas easier to understand. If needed, search Excel's Help for "names in formulas."

Next, we incorporate randomness. Excel draws uniformly distributed random numbers in the interval from zero to one with the RAND () function.

$S T E P$ In cell A3, enter the formula $=R A N D($ ).

You see a number with several decimal places displayed that is between zero and one. The number is actually much longer.

$S T E P$ Widen the column and add decimal places to see this. Keep adding decimal places (widening column A as needed) until you start seeing zeroes.

Most modern spreadsheets use 64-bit double-precision floating point format which is roughly like 16 significant decimal digits. If you count carefully, you will see that $\operatorname{RAND}()$ has a zero, then a decimal point, and then 15 decimal places with values from zero to nine. After that, they are all zero so we have reached the maximum precision. It is important to understand that our spreadsheet's random number is finite, but also that it has many more decimal digits than what was originally displayed.

STEP Repeatedly press F9. This is the keyboard shortcut to recalculate the sheet.

The number in cell A3 changes each time you recalculate the sheet. This is incredibly powerful because we now have a random number generator that will allow us to model random infection in a population.

$S T E P$ In cell A4, enter the formula $=I F(A 3<$ InfectionRate, 1, 0). 
The IF function has three arguments (or inputs), separated by commas. The first argument is the test, the second is what happens if the test is true (or yes), and the third is what happens if it is false (or no). If the random number in cell A3 is less than 5\%, then cell A4 shows a one, which means the person is infected; otherwise, it shows a zero, which means they are not infected.

Some students (usually really smart, careful ones) obsess about whether A3 should be less than $(<)$ or less than or equal to $(\leq)$ the infection rate. This does not matter because the random number drawn is so small. The chances of drawing exactly 0.050000000000000 are ridiculously small.

$S T E P$ Press F9 repeatedly to recalculate the sheet until you see a one in cell A4. The chances are only 1 in 20 so be patient.

As you recalculated, cell A3 constantly changed, but cell A4 changed only if A3 switched from being above or below the infection rate. Cell A4 is a binomial random variable because it can take only the values zero or one.

Now that we know how to implement a random process that outputs whether or not an individual is infected, we can create an entire population of people, some with the virus and others without it.

\section{$S T E P$ In cell C1, enter the formula $=\operatorname{IF}(R A N D()<$ InfectionRate, 1,0$)$.}

Notice how we directly embedded the RAND() function in the cell formula. We do not know which random number was drawn, but we do know if it was less than $5 \%$ because then cell $\mathrm{C} 1$ would be one.

$S T E P$ Fill down this formula all the way to cell C1000. If needed, search Excel's Help for "fill down."

As you scroll back up to the top row, you will see a sprinkling of ones among many zeroes. With a $5 \%$ infection rate, roughly one in twenty cells will have random number draws less than $5 \%$ and, therefore, show a one.

The fact that each cell in column $\mathrm{C}$ stands alone and does not depend on or influence other cells means we are assuming independence. In our model, one person with the virus does not affect the chances of anyone else being infected. If this condition is violated, then our model and results are compromised. We would make the chances of infection depend on whether friends and family have the virus, for example, to improve our analysis. 
How many people in our population of 1,000 are infected?

$S T E P$ Enter the formula $=S U M(C 1: C 1000)$ in cell D1 and the label, "Number infected" in cell E1.

You will see a number around 50 in cell D1. The number of infected people is not always exactly 50 because there is chance involved in who gets infected.

$S T E P$ Use your keyboard shortcut to recalculate the sheet a few times to get sense of the variability in the number of infected people.

The total number of infected people can be less than 40 or more than 60 , but that is not common. There is no doubt, however, that the number of infected people is a random number since it is bouncing around when you recalculate the sheet. It makes common sense that adding binomial random variables will produce a random outcome.

We can make it easier to identify who is infected with a spreadsheet's conditional formatting capability. This offers the viewer visual cues that make data easier to understand.

STEP Select the entire column $\mathrm{C}$ and apply a formatting rule that highlights, with color, cells with a value of one. Choose font and fill colors that you think emphasize being infected. If needed, search Excel's Help for "conditional formatting."

Now, when you scroll down, it is easy to see who is infected. Recalculation changes who is infected - it is as if we rewound and replayed the world.

Having implemented the chance process for having the virus or not, we turn to pooled testing. Instead of testing each person, we can group individuals and test the group. If the group tests positive, then we know at least one person is infected; if not, we know no one is infected and we do not have to test each individual in the group.

We can choose the size of the group and this will determine how many groups we have since

$$
\text { NumberofGroups }=\frac{\text { Population }}{\text { GroupSize }}
$$


With our population of 1,000 people, a group size of 100 means we will have 10 groups. Intuitively, with an infection rate of 5\%, 100 people in a group means that the group is probably going to test positive. We can make our intuition more convincing by computing the exact chances.

$S T E P$ Begin by entering 100 in cell D3 and the label "Group Size" next to it in cell E3. Enter the formula $=1000 / D 3$ in cell D4 and the label "Number of Groups" in cell E4.

An infection rate of $5 \%$ means each person has a $95 \%$ chance of not being infected. If there are two people (assuming the chances of infection are independent), then there is a $0.95 \times 0.95=0.95^{2}=0.9025$, or $90.25 \%$, chance that neither are infected. This means there is a $100 \%-90.25 \%=9.75 \%$ chance that at least one of the two people is infected.

What are the chances that at least one person is infected in a group of 100 people? Remember, if even one person is infected in a group, we have to test everyone in the group to find out who is infected.

$S T E P$ In cell D6, enter the formula $=(1-\text { InfectionRate })^{\wedge} D 3$ and the label "prob no one in the group infected" in cell E6. Format D6 as a percentage so that it displays $0.59 \%$.

But this is the probability that no one is infected in the group. We subtract this from one to find the likelihood that at least one person is infected.

$S T E P$ In cell D7, enter the formula $=1-D 6$ and the label "prob at least one in the group infected" in cell E7. Format D7 as a percentage (if needed).

With an infection rate of $5 \%$, doing pooled testing with a group size of 100 is wasteful. After all, it seems overwhelmingly likely (over 99\%) that we will have to test everyone in each of the ten groups so we would end up doing 1,010 tests.

Can we make our spreadsheet show how many people are infected in each group and confirm the computations we just made? We can, but the approach described below uses a function which may be unfamiliar - the OFFSET reference function. Thus, we proceed slowly.

$S T E P$ In cell G1, enter the formula $=\operatorname{OFFSET}(E 1,3,0)$. 
Cell G1 computes the number of groups because the OFFSET function went to cell E1 (the first argument in the function), then went three rows down (the second argument). The third argument is zero, so it stayed in column E. If the movement arguments are a positive integer we move down or right; negative integers move us up or left.

\section{$S T E P$ Change the formula in cell G1 to $=\operatorname{SUM}(\operatorname{OFFSET}(D 1,0,0,3,1))$.}

Why does G1 display D1 plus 100? The two zeroes means it did not move from the reference cell D1, but the fourth and fifth arguments control the height and width, respectively, of the cell range. Therefore, the formula says to add up the values in cells D1, D2 (which is blank), and D3 (100).

We want to add up the values in column $\mathrm{C}$ into ten separate groups of 100 each. We can modify our OFFSET function to do the first group of 100 .

$S T E P$ Change the formula in cell G1 to $=\operatorname{SUM}(\operatorname{OFFSET}(C 1,0,0,100,1))$.

The value reported in cell G1 is the sum of the first 100 people in the population. How can we get the second group of 100 people?

$S T E P$ Change the formula in cell G1 to $=\operatorname{SUM}(\operatorname{OFFSET}(\$ C \$ 1,0,0,100,1))$ and fill it down to cell G2.

Adding the dollar signs made it an absolute reference so we kept our C1 starting point in cell G2, but we need to change the formula so it adds up the number of infected people in the second set of 100 . We do that by changing the second argument because it controls how many rows to move from the reference cell.

$S T E P$ Change the formula in cell $\mathrm{G} 2$ to $=\operatorname{SUM}(\operatorname{OFFSET}(\$ C \$ 1,100,0,100,1))$.

Cell G2 reports how many people are infected in the second group of 100 . We could fill down eight more cells and then change the second argument manually to 200,300, and so on, but this is poor spreadsheet practice. You never want to manually re-enter the same thing or an ordered sequence of numbers.

In addition, you want to maximize flexibility. Hard-coding numbers, like 100, in formulas is poor practice because you might want to change that number in the future. 
In this case, we want our groupings to respond to changes in cell D3. If, for example, we have a group size of 50, we would then have 20 groups. We want the spreadsheet to automatically show how many people are infected in each of the 20 groups.

This task requires that we modify the second and fourth arguments. The fourth argument is the group size, which is simply cell D3. The second argument is more complicated. It is zero for the first group, then increases by D3 for each group.

One way to do this is to use the ROW function, which returns the row number of a cell.

$S T E P$ In cell G2, enter the formula $=\operatorname{ROW}(D 6)$.

Cell G2 displays 6, the row number of cell D6. What happens if the ROW function does not have an argument?

$S T E P$ Change the formula in cell G2 to $=R O W()$ and fill it down to G10.

Without an argument, the ROW function returns the row number of the cell which contains ROW() in the formula. We can use this to create a series that starts at zero and increases by the amount in cell D3.

$S T E P$ Change the formula in cell G2 to $=(R O W()-1) * \$ D \$ 3$ and fill it down to G10.

We can use our ROW function strategy in the OFFSET function's second argument to create a formula that gives us the number of infected people for any group size from 2 to 500 entered in D3. A "group" of one is simply individual testing and with 1,000 people, a group of size two yields 500 groups.

We start with G1 (notice that ROW()-1 is zero for G1 so the second argument evaluates to zero) and fill down to G500 (since 500 is the maximum number of groups we can have).

$S T E P$ Change the formula in cell G1 to $=\operatorname{SUM}(\operatorname{OFFSET}(C 1,(\operatorname{ROW}()-1)$ $* \$ D \$ 3,0, \$ D \$ 3,1))$ and fill it down to G500.

Cells G1 to G10 now display the number of infected people in each of the ten groups of 100 people. 
STEP Click the letter $\mathrm{C}$ in column $\mathrm{C}$ to select the entire column, and then click the Format Painter button (in the Home tab in the ribbon, or top menu). Now click the letter $G$ in column G.

You applied the formatting in column $\mathrm{C}$, including your conditional formatting to highlight the infected people, to column G. It (probably) shows all of the groups highlighted, but it will soon come in handy when we lower the group size so some groups have no infected people.

It is good practice to include checks in your spreadsheets. In this case, an easy check is to see if the sum of infected people in the ten groups equals the total number of infected people in the population in column $\mathrm{C}$.

$S T E P$ In cell H1, enter the formula $=S U M(G 1: G 500)$ and the label "check" in cell I1, then recalculate the sheet a few times.

It is easy to see that cells D1 and H1 are the same. If not, something is wrong. STEP Change cell D3 to 200 and recalculate the sheet a few times.

Now only five cells in column $\mathrm{G}$ have nonzero values, representing the number of infected people in each of the five groups.

Group sizes of 100 and 200 are way too big because we are extremely unlikely to get a group where everyone tests negative so we almost always have to test everyone in the group.

$S T E P$ Change cell D3 to 20 and recalculate the sheet a few times.

Now we are really getting somewhere. Column $\mathrm{G}$ is showing the number of infected people in each of 50 groups. You can see values of $0,1,2,3$, and, less frequently, higher numbers. We love to see zeroes because they mean we do not have to test anyone in that group and we saved 20 tests.

How many tests will we have to run in total? The COUNTIF function allows us to count the number of cells in a range that meet a specific condition.

$S T E P$ In cell H2, enter the formula $=\operatorname{COUNTIF}(G 1: G 500, ">0$ ") and the label "number of groups to test" in I2. 
The COUNTIF function reports the number of cells in the range G1:G500 that have a value greater than zero. If we multiply this by the group size, we know how many individual tests we have to run. This is added to the number of group tests to give us our total number of tests.

$S T E P$ In cell H3, enter the formula $=H 2{ }^{*} D 3$ and the label "tests from infected groups" in I3. In cell H4, enter the formula $=\mathrm{D}_{4}+\mathrm{H}_{3}$ and the label "total tests" in I4.

Notice that, once again, we did not hard-code numbers (like 20 for group size) into the formula. We want our spreadsheet to respond to changes in group size (cell D3) automatically.

Cell H4 is certainly giving us good news. Total tests is a random variable that is almost certainly less than 1,000. You are likely to see numbers around 690 tests, give or take 70 or so. This is about a $30 \%$ decrease in the number of tests from the 1,000 required by individual testing.

\section{Finding the Optimal Group Size}

We have come a long way in that we have implemented a chance process of getting infected in our spreadsheet and demonstrated the power of pooled testing. Grouping allows us to save on testing because any groups that have no infected people means we do not have test those individuals.

Our spreadsheet shows that a group size of 20 is better than individual testing, but we do not want to do merely better than 1,000 tests. We want to perform the fewest number of tests. Our fundamental question is: What is the optimal group size?

There is a complication that we have to confront to answer our question: total tests is a random variable. We could get lucky and have a population that has fewer than 50 infected people that happen to be more clumped together than usual so that lots of groups have people without the virus. This would mean we would have to run fewer tests than usual.

We deal with the fact that total tests is a random variable by focusing on the expected value of total tests. This is what we would typically observe. We need to find the expected value of total tests for a given group size so we can figure out which group size minimizes it. 
There are mathematical rules for computing the expected value, but we will use an alternative approach called Monte Carlo simulation. It is based on the idea that we can simply run the chance process (throwing two dice or hitting F9) many times and then compute the average outcome. This provides an approximation to the expected value.

So, we seek the group size that minimizes the expected value of total tests, which we will approximate by simulation. We will run many repetitions (recalculating the sheet repeatedly) and keep track of the total number of tests to see how many total tests we can expect to run as we vary group size.

While there are many simulation add-ins available for Excel, we can easily run a simulation using Excel's Data Table tool. It was not designed to run a simulation, but to display multiple outcomes as inputs vary. To do this, it recalculates the sheet, which enables us to perform Monte Carlo analysis.

STEP In cell L1, enter the number 1 and enter 2 in cell L2. Select both cells and fill down to row 400 so that you have a series from 1 to 400 in column L.

Next, we provide the cell that we wish to track, total tests.

$S T E P$ In cell M1, enter the formula $=H_{4}$.

We are now ready to create the Data Table.

STEP Select the cell range L1:M400, click the Data tab in the ribbon, click What-If Analysis in the Forecast group, and select Data Table... A keyboard shortcut is alt-a-w-t.

Excel pops up the Data Table input box.

$S T E P$ Click in the column input cell field, click on cell $\mathrm{K} 1$, and click $O K$.

Clicking on an empty cell would be meaningless if we were using the Data Table tool for its intended purpose, which is to show how an input cell affects a formula in another cell. All we want, however, is for Excel to recalculate the sheet and show us the total tests for that newly recalculated population in column $\mathrm{C}$. 
The display in column M shows 400 repetitions of hitting F9 and keeping track of total tests. This is exactly what we want because now we can take the average of the total tests values to approximate the expected number of total tests when the group size is 20 .

But before we do this, let's be clear about what a Data Table is actually doing. Be aware in the next step, however, that if you double-click on a cell in column $\mathrm{M}$ or click in the formula bar, you might get trapped in a cell. If you get stuck, press the esc (escape) key to get out.

$S T E P$ Click on a few cells from M2 to M400 to see that they have an array formula: $\{=T A B L E(, K 1)\}$.

Excel gives users a friendly front-end via Data: What-If Analysis: Data Table... to create an array formula (indicated by the curly brackets, \{\} ) that can display multiple outputs. You cannot change or delete an individual cell in the range M2:M400. They are, in a sense, a single unit, sharing the same formula.

You might also notice that the sheet is much slower as we enter formulas or press F9. This is due to the Data Table. Excel now has many more cells to recalculate and evaluate. We could do many more repetitions (usually simulations have tens of thousands of repetitions), but the delay in recalculation is not worth it. With 400 repetitions, the approximation is good enough for our purposes.

$S T E P$ In cell N1, enter the formula $=A V E R A G E(M 1: M 400)$ and the label "approximate expected value of total tests" in cell O1.

Cell N1 is our simulation's approximation to what we want to minimize. It gives us a handle on the center of the sampling distribution of the statistic, totals tests. A statistic is a recipe for what to do with observations (in this case, given by the formula in cell $\mathrm{H} 4$ ). If we make a histogram of the data in column $\mathrm{M}$, we get an approximation to the sampling distribution of total tests.

Excel 2016 or greater is needed to make the histogram chart. This is not the Histogram option in the Data Analysis add-in (from the Analysis Tool-Pak). The histogram chart allows for dynamic updating and is a marked improvement over the histogram in the Data Analysis add-in. 
$S T E P$ Select cell range M1:M400, click the Insert tab in the ribbon, and select Histogram from the Charts group. It is in the Statistic chart group and, of course, in the collection of all charts (available by clicking the bottomright corner square in the Charts group).

The default bin widths are a little too big, but they are easy to adjust.

$S T E P$ Double-click the chart's $x$ axis and in the Axis Options, set the Bin Width to 20. Make the title "Approximate Sampling Distribution of Total Tests."

The chart is an approximation because it is based on only 400 repetitions. The exact sampling distribution of total tests would require an infinite number of repetitions. We can never get the exact sampling distribution or the exact expected value via simulation, but the more repetitions we do, the better the approximation.

Even with just 400 realizations of total tests, the graph looks a lot like the classic, bell-shaped distribution of the normal (or Gaussian) curve. The center is the expected value and the dispersion is measured by the standard error.

$S T E P$ In cell N2, enter the formula $=S T D E V . P(M 1: M 400)$ and the label "approximate standard error of total tests" in cell O2.

The standard error of total tests tells us the variability in total tests. It is a measure of the size of the typical bounce in total tests.

$S T E P$ Press F9 a few times and watch cell H4.

You can see H4 is bouncing around. In fact, it is centered around 690 and jumps by roughly plus or minus 70 total tests as you hit F9. You can also scroll up and down column $\mathrm{M}$ to see that the total tests numbers are around $690 \pm 70$.

Simulation can not give us the exact standard error, but the standard deviation of our 400 realizations of total tests is a good approximation of the standard error.

There are many ways to be confused here. One of them is to fixate on the computation of the standard deviation. We used STDEV.P (for population) 
instead of STDEV.S (for sample) because we are not using the standard deviation to estimate the population standard deviation so we do not need to make a correction for degrees of freedom. Although the population standard deviation is correct, this makes almost no difference with 400 numbers.

$S T E P$ In cell N3, enter the formula $=S T D E V . S(M 1: M 400)$ and compare the result to cell $\mathrm{N} 2$.

The Great Debate over population versus sample standard deviation in many Statistics courses is only relevant for small sample sizes, say less than $30 \mathrm{ob}-$ servations. As the number of observations rises, the two grow ever closer.

To summarize, cells N1 and N2 tell us that we can expect to perform about 690 total tests, give or take roughly 70 tests. These are the numbers reported at the end of the previous section. This is for a group size of 20. Can we do better? We get to choose the group size so we should explore how the expected number of total tests responds as we vary group size.

STEP Change the group size (in cell D3) to 10 and press F9 a few times. Which specific cell should you focus on and what do you conclude?

The cell we care about the most is cell N1 because it tells us (approximately) the expected number of tests we will have to run. Cell N1 is reporting good news. We can expect to perform about $500 \pm 50$ total tests. That beats the group size of 20 by almost 200 tests, on average, and is a large saving of half versus 1,000 individuals tests.

Why does a group size of 10 do better than 20? There is evidence in various cells of the spreadsheet of what is happening. Lowering the group size from 20 to 10 increased the number of group tests from 50 to 100 (see cell D4), but the number of infected groups only went up a little bit (from roughly 32 to 40 ) and the groups are now much smaller. This is where the big savings are-instead of $32 \times 20=640$ tests, we only have to run, on average, $40 \times 10=400$ tests with a group size of 10 .

$S T E P$ Confirm the claims above by switching back and forth from 10 to 20 in cell H4. Press F9 and read the various cells and the chart.

Spreadsheets are powerful because they can display a lot of information and dynamically update when you make changes. Your job is to make comparisons and process the information. 
Can we do even better than a group size of $10 ?$

$S T E P$ Change the group size (in cell D3) to 5.

Amazing! Cell N1 fell again. The expected number of total tests is now about 425 (426.22 is a more exact answer, found by analytical methods) give or take roughly 30 tests. That is a gain of almost $60 \%$ versus individual testing. Pooled testing saves a lot of tests compared to individual testing.

As before, the number of groups we have to test has risen (this time to 200), but many groups are found to be uninfected. Cell D6 reports a $77.4 \%$ chance that no one in a five-person group will be infected. Thus, even though we test more groups, we more than make up for this because many groups test negative, saving us the need to test five people in the group.

The group size of 5 is, in fact, the optimal solution and answer to our question. Figure 1 reveals that we traveled down the expected number of total tests curve as we changed group size from 20 to 10 and finally 5 .

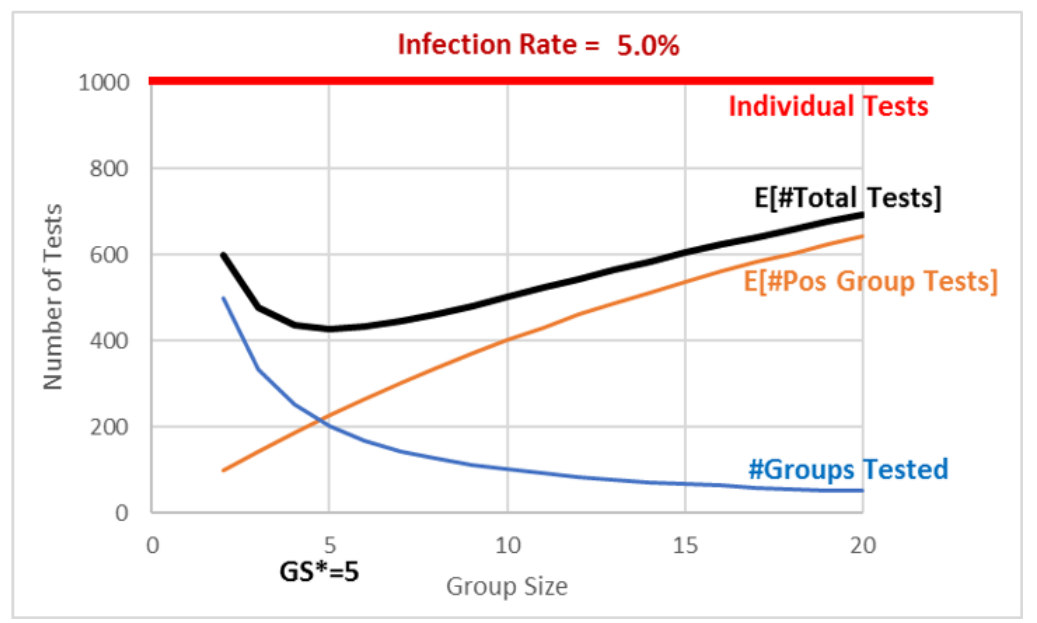

Figure 1: The expected number of tests as a function of group size.

Figure 1 makes it easy to see that a group size of five is the minimum of the expected number of total tests curve, but it also reveals the trade-off involved. The two curves are added up to produce the top, total curve. At 10, we test 100 groups (the bottom curve) and we add that to 400 (the expected number of tests from positive groups) and this gives 500 (the top curve). 
When we moved from 10 to 5, we added 100 tests (the bottom curve), but saved about 175 tests (the middle curve), lowering our expected total tests from 500 to 425 . We cannot do any better than 425 total tests. Further reduction in group size will increase total tests.

\section{Comparative Statics Analysis}

We can ask another question which, again, shows off the power of spreadsheets: what happens if the infection rate changes, say to $1 \%$ - what would be the optimal group size?

This kind of question is called comparative statics analysis because we want to know how our solution responds to a shock. We want to compare our initial, optimal group size of five when the infection rate was $5 \%$, to the new solution when the infection rate is $1 \%$. This comparison reveals how the shock (changing the infection rate) affects the optimal response (group size).

$S T E P$ Change cell A1 to $1 \%$, then use the spreadsheet to find the optimal group size. What group size would you recommend? Why?

You may have struggled with this because it turns out that the total tests curve is rather flat at its minimum. Thus, a simulation with 400 repetitions does not have the resolution to distinguish between group sizes in the range from 8 to 14 or so. Figure 2 makes this clear.

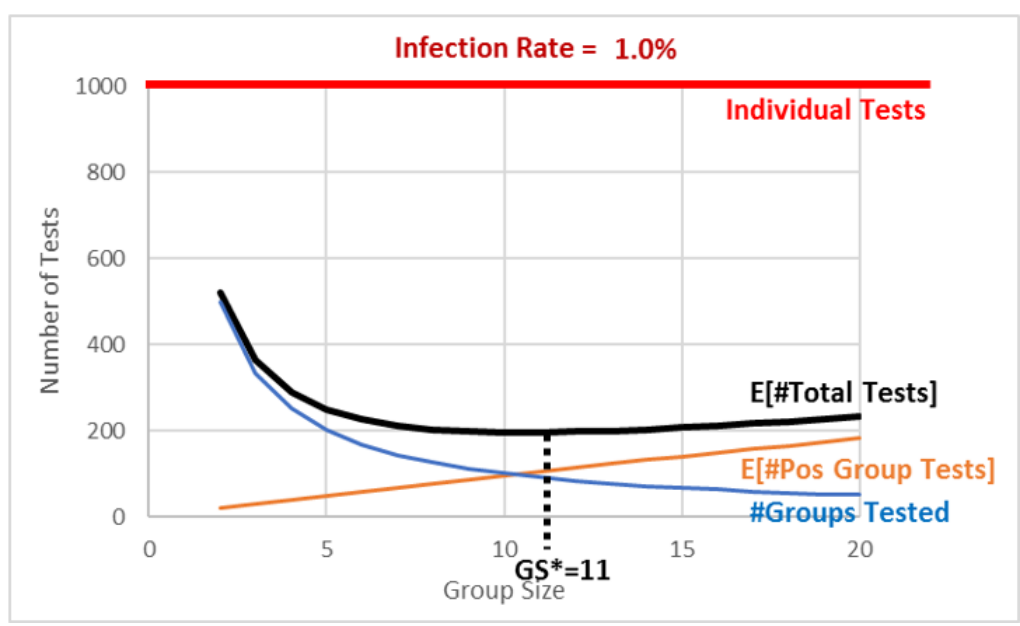

Figure 2: Optimal group size with a $1 \%$ infection rate. 
The exact answer for the optimal group size is, in fact, 11 groups. It has an expected number of total tests of 195.57 (again, using analytical methods). Choosing group sizes of 10 or 12 lead to a slightly higher number of total tests - although it is impossible to see this in Figure 2.

An infection rate of $1 \%$ shows that Monte Carlo simulation may not be an effective solution strategy for every problem. Of course, you could have created a Data Table with more repetitions, but using simulation to distinguish between group sizes 10 and 11 requires a Data Table so large that Excel would be unresponsive.

As mentioned earlier, there are many Excel Monte Carlo simulation add-ins and they can do millions of repetitions. A free one is here: tiny.cc/mcsim. Even if we recalculated enough times to see that 11 is the answer, you should remember that simulation will never give you an exact result because it can never do an infinity of repetitions.

The good news is that any group size around 10 is going to be a little under 200 which is an $80 \%$ improvement over individual testing. There is no doubt about it - pooled testing can be a smart, effective way to reduce the number of total tests performed.

Our comparative statics analysis tells us that the lower the infection rate (from $5 \%$ to $1 \%$ ), the bigger the optimal group size (from 5 to 11 ) and the greater the savings from pooled testing versus individual testing (from about 675 to 800 tests).

Finally, if you carefully compare Figures 1 and 2, you will see that the \#Groups Tested curve (it is a rectangular hyperbola since the numerator is constant at 1,000) stays the same in both graphs. Changing the infection rate shifts down the E[\#Pos Group Tests] relationship and this brings down and alters the shape of the E[\#Total Tests] curve.

This comparative statics analysis has shown that pooled testing is more effective when the infection rate falls. A lower infection rate means we can have bigger groups and yet they may still have no infected individuals in them. 


\section{Takeaways}

Figure 3 shows a list of the Excel skills, functions, and concepts covered in creating the pooled testing spreadsheet.

By creating this spreadsheet, you have improved your Excel skills and confidence in using spreadsheets. You added to your stock of knowledge that will help you next time you work with a spreadsheet.

\begin{tabular}{|llll|}
\hline \multicolumn{1}{|c}{ Excel Skills } & Excel Functions & \multicolumn{1}{c|}{ Concepts } \\
\cline { 2 - 3 } Naming cells & RAND() & Random number generation \\
Display and Precision & IF(test, true, false) & Binomial random variable \\
Arguments in a function & SUM(range) & Independence \\
Fecalculation (F9) & OFFSET(ref, rows, col, height, width) & Probability computation \\
Conditional formatting & ROW() & Expected value \\
Format Painter & COUNTIF(range, condition) & Monte Carlo simulation \\
Checking results & STABLE(,)\} -- Data Table array & Sampling Distribution \\
Flexibility (no hard-coding numbers) & AVERAGE(range) & Standard error (variability in a statstic) \\
Histogram chart (requires Excel 2016 or greater) & STDEV.P(range) and STDEV.S(range) & Optimization (minimization) \\
Array function (TABLE) & & Comparative statics analysis \\
\hline
\end{tabular}

Figure 3: Topics covered.

You also learned or reinforced a great deal of statistical and economics concepts. Economics has a toolkit that gets used over and over again-look for similar concepts in future models and courses. Try to spot the patterns and repeated logic. Although it may not be explicitly stated, getting you to think like an economist is a fundamental goal of almost every Econ course.

One methodology issue that is easy to forget, but crucial, is that we made many simplifying assumptions in our implementation of the data generation process. There may be other factors at play in the spread of covid-19 or how tests actually work that affect the efficacy of pooling. For example, Wu (2020) says that, "A positive specimen can only get diluted so much before the coronavirus becomes undetectable. That means pooling will miss some people who harbor very low amounts of the virus." 
Our results apply to an imaginary, perfect world, not the real world. We need to be careful in moving from theory to reality. This requires both art and science.

The introduction cited Dorfman as writing a paper on pooled testing back in 1943. It is a clever idea that you now understand can be used to greatly reduce the number of tests, which saves a lot of resources. Perhaps you will not be surprised to hear that Robert Dorfman was an economist.

\section{References}

Dorfman, R. (1943) "The Detection of Defective Members of Large Populations," Ann. Math. Statist. 14, no. 4, 436-440, projecteuclid.org/euclid. aoms/1177731363

Mandavilli, A (2020) "Federal Officials Turn to a New Testing Strategy as Infections Surge," The New York Times, July 1, 2020,

www.nytimes.com/2020/07/01/health/coronavirus-pooled-testing.html

Wu, K (2020) "Why Pooled Testing for the Coronavirus Isn't Working in America," The New York Times, August 18, 2020, www.nytimes.com/2020/08/18/health/coronavirus-pool-testing.html 